\title{
Minimally Invasive Treatment for Hard Palate-Invading Maxillary Keratocystic Odontogenic Tumor
}

\author{
Kazuhiro Nomura, ${ }^{1,2}$ Kazuya Arakawa, ${ }^{1}$ Fumiyoshi Fujishima, ${ }^{3}$ Yuto Yamazaki, ${ }^{3}$ \\ Daiki Ozawa, ${ }^{1}$ Yuri Nomura, ${ }^{1}$ Hiroshi Hidaka, ${ }^{1}$ Naohiro Yoshida ${ }^{2}$ and \\ Yukio Katori ${ }^{1}$ \\ ${ }^{1}$ Department of Otolaryngology-Head and Neck Surgery, Tohoku University Graduate School of Medicine, \\ Sendai, Miyagi, Japan \\ ${ }^{2}$ Department of Otolaryngology, Jichi Medical University, Saitama Medical Center, Saitama, Saitama, Japan \\ ${ }^{3}$ Department of Pathology, Tohoku University Graduate School of Medicine, Sendai, Miyagi, Japan
}

\begin{abstract}
Keratocystic odontogenic tumor (KCOT) is one of the benign developmental odontogenic cystic lesions arising from impacted teeth. In comparison to other odontogenic cysts, such as radicular cysts and dentigerous cysts, KCOT is known to be more aggressive and is associated with a relatively high recurrence rate. Traditionally, KCOT has been treated with total resection through sublabial incision. Marsupialization is advocated to reduce surgical invasion. However in all the cases, marsupialization was performed in the oral cavity. With the recent development of appropriate instruments and the endoscopic modified medial maxillectomy (EMMM) technique, which allows preservation of the inferior turbinate and nasolacrimal duct, an exclusive endoscopic approach to KCOT becomes possible. However, when the KCOT invades the hard palate, total resection of the tumor requires subtotal maxillectomy including hard palate. Consequently, as the maxillary sinus connects to the oral cavity, life-long use of a prosthesis becomes mandatory. Here we report a case of a seventeen-year-old female with a hard palate-invading KCOT who was successfully treated with the EMMM approach. The KCOT was fenestrated to the nasal cavity, leading to preservation of the hard palate. The lesion invading the hard palate was found to remain unchanged over one year upon follow-up. The trans-nasal approach with EMMM is a direct, minimally invasive method providing a direct field of view for the treatment of maxillary odontogenic cysts. Marsupialization of the KCOT with the EMMM technique might be a viable treatment option if the maxillary KCOT invades surrounding structures.
\end{abstract}

Keywords: endoscope; marsupialization; medial maxillectomy; odontogenic cyst; surgery

Tohoku J. Exp. Med., 2015 December, 237(4), 267-272. C 2015 Tohoku University Medical Press

\section{Introduction}

Keratocystic odontogenic tumor (KCOT) is a benign odontogenic cystic lesion with a characteristic lining of parakeratinized stratified squamous epithelium, and are most common in the second and third decades of life (Barnes et al. 2005). Compared to other odontogenic cystic lesions, KCOT has locally destructive characteristics and is associated with a high recurrence rate after surgery (Vered et al. 2005; Giuliani et al. 2006; Habibi et al. 2007; Cakur et al. 2008; González-Alva et al. 2008; Grasmuck and Nelson 2010).

Traditionally, the treatment for KCOT has been total resection and extraction of the cyst-associated tooth (Habibi et al. 2007; González-Alva et al. 2008; Grasmuck and Nelson 2010). The recurrence rate with total resection var- ies among reports. Bataineh and al Qudah (1998) reported $0 \%$ of recurrence rate in their own 31 cases of mandibular KCOT but review of the literature has indicated that recurrence rates may vary from 0 to $62 \%$. The high recurrence rate then led surgeons to believe that that complete removal of the KCOT with meticulous curettage of surrounding tissues was necessary in order to address the problem of high recurrence. Marsupialization for KCOT to minimize surgical invasion has been reported (Nakamura et al. 2002; Pogrel and Jordan 2004; Maurette et al. 2006), however, all marsupializations were performed in the oral cavity. Recently, other surgical strategies have been advocated. Endoscopic modified medial maxillectomy (EMMM) with preservation of the inferior turbinate and nasolacrimal duct was proposed for the treatment of inverted papilloma of the anterior wall of the maxillary sinus (Nakayama et al. 2012).

Received September 10, 2015; revised and accepted October 14, 2015. Published online November 14, 2015; doi: 10.1620/tjem.237.267.

Correspondence: Kazuhiro Nomura, M.D., Department of Otolaryngology, Jichi Medical University, Saitama Medical Center, 1-847 Amanuma, Omiya-ku, Saitama, Saitama 330-8503, Japan.

e-mail: nomura@jichi.ac.jp 
Subsequently, the same authors reported that this EMMM approach was also useful for treating odontogenic maxillary cysts (Nakayama et al. 2014). In all of their cases, the cysts were restricted to the maxillary sinus and could be removed. Currently, the treatment option for maxillary KCOTs that invade the hard palate remains to be elucidated.

Here we describe the course of a hard palate-invading KCOT treated with marsupialization using the EMMM surgical approach, which demonstrated no progression of the lesion after being followed-up for more than one year.

\section{Case Presentation}

A 17-year-old female who presented with a sense of unusual smell and nasal obstruction was referred to our department. She observed nasal obstruction on the right side that was accompanied by a foul odor, and had visited a local hospital one month before presentation at our department. She had no history of maxillofacial trauma, genetic or other immuno-compromising disorders. Axial and coronal computed tomography (CT) showed opacification of right maxillary sinus with a tooth attached to posterior wall of the maxillary sinus (Fig. 1A-C). Three-dimensional reconstruction $\mathrm{CT}$ imaging demonstrated a molar-shaped tooth erupting from the posterior maxillary wall (Fig. 1D). She was transferred to our department for surgical interven- tion. Since the thin bony cyst wall was observed on $\mathrm{CT}$ imaging (Fig. 1A, C, arrow), dentigerous cyst was suspected preoperatively. Thus, in case complete removal of the cyst was impossible without external approach, marsupialization of the cyst was presumed to be surgical option. Endoscopic sinus surgery was performed under general anesthesia. First, a middle meatal antrostomy was made. Cyst wall was paper like bone and was easily penetrated. Cloudy yellow fluid filled the cyst. Debris was not observed. The tooth was too firm to extract with curved instruments (Fig. 2A). The EMMM approach was then utilized. An perpendicular incision was made in the lateral nasal wall at the level of anterior tip of the inferior turbinate (Nakayama et al. 2012). The complex of the nasolacrimal duct, mucosal flap, and inferior turbinate was pushed medially and preserved. The medial bony wall of the maxillary sinus was then drilled with a diamond burr. The posterior wall of the maxillary sinus was now in the center of the field of vision (Fig. 2B). The tooth was extracted with a large straight cup forceps (Fig. 2C). Most of the cyst wall was removed, but the portion attaches to the maxillary floor and the mucosa with inflammation change at the lateral part was left untouched. With inflammation, mucosa and surrounding soft tissue was not distinguishable. Bleeding was minimal. Finally, the inferior turbinate, nasolacrimal duct,

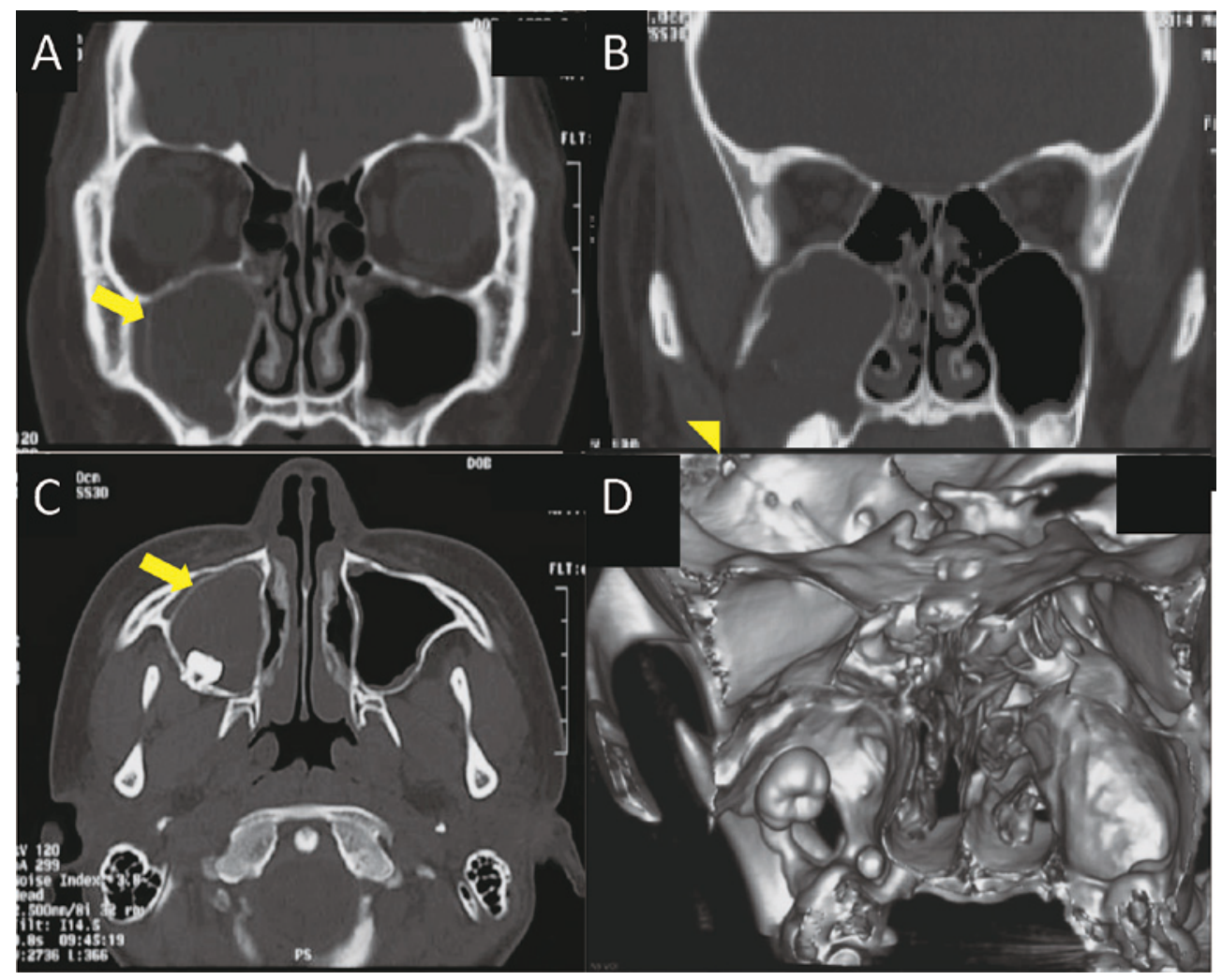

Fig. 1. Preoperative plain computed tomography.

Coronal (A, B) and axial (C) views demonstrating the opacified right maxillary sinus. Cyst wall (arrow) can be seen on (A) and (C). Infero-lateral part of maxillary bone is absorbed (arrow head) (C). A tooth has erupted from the posterior maxillary wall $(\mathrm{C})$. A three-dimensional reconstructive image shows a molar-like shaped tooth erupting from the posterior wall (D). 


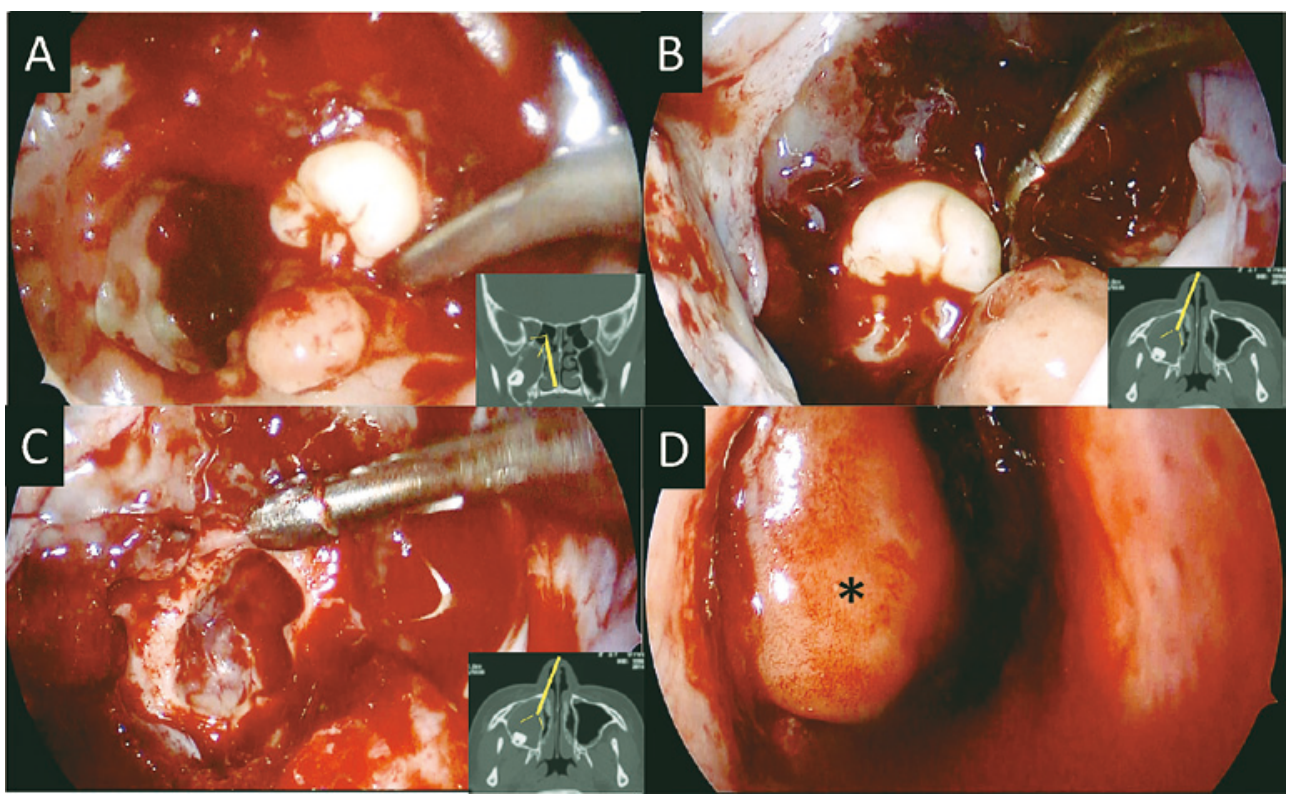

Fig. 2. Intraoperative photographs.

The direction of the endoscope is shown in insets. (A) View with 70 degree endoscope. The tooth in the maxillary sinus cannot be removed with a 120 degree curved instrument (\#18-83517, Medtronic, Jacksonville, FL). (B) View with 0 degree endoscope through EMMM approach. The tooth attached to the posterior maxillary wall can be seen at the center of the field of vision. (C) View with 0 degree endoscope through EMMM approach. After the tooth was extracted. (D) View with 0 degree endoscope. Entire inferior turbinate $(*)$ was preserved after the operation.

and lateral mucosal wall of the inferior meatus were replaced (Fig. 2D). No fistula was made at the inferior meatus. The pain remitted soon after the surgery. Histological study showed cystic lesion whose lumen was lined by the parakeratinized thickened squamous epithelium. Mitotic figures and atypical mitotic figures were not recognized. Inflammatory cells, including plasma cells, lymphocytes and histiocytes intensively infiltrated within the fibrous stroma. These pathological findings were compatible with KCOT (Fig. 3). Follow up magnetic resonance imaging (MRI) showed that the lesion at the hard palate was stable one year after the operation (Fig. 4).

\section{Discussion}

KCOT was previously referred to as an odontogenic keratocyst. As it is associated with an aggressive characteristic, a high recurrence rate, and based on histological findings, the term was changed to KCOT in the WHO classification in 2005 (Barnes et al. 2005; Cakur et al. 2008). For a long time, total resection was the only treatment option for KCOT (Habibi et al. 2007; González-Alva et al. 2008; Grasmuck and Nelson 2010), but complete removal of a large KCOT requires an extensive approach. Some surgeons adopt this conservative approach, which is total resection, for KCOT, whereas marsupialization followed by close follow-up has been proposed by other groups (Nakamura et al. 2002; Pogrel and Jordan 2004; Maurette et al. 2006; Habibi et al. 2007). Outcomes with marsupialization alone were extremely favorable. Most of the tumors diminished or regressed. However in all of the cases, the
KCOT was marsupialized through the oral cavity and this may result in discomfort for the patient. In contrast, Ohki (2012) reported endoscopic transnasal marsupialization and removal of the tooth for maxillary KCOT, and this was associated with no progression within a one year follow up. In his case, the tooth erupted from the posterior-medial corner of the maxillary sinus where it is possible to remove the tooth. However, in our case the tooth was located at the inferior portion of the posterior wall of the maxillary sinus and could not be removed through middle meatal antrostomy due to the lack of torque. Therefore we employed the EMMM approach. With this approach, straight instruments could reach all sections of the posterior wall of the maxillary sinus. With large straight cup forceps, the tooth could then be removed.

Recently, Menon (2015) published a review paper on etiology, pathogenesis and treatment of KCOT. He concluded that total resection should be limited to large lesions affecting most of the bony structure. Conservative treatment is preferable. Long term follow up for at least 5 years is needed. He proposed using Carnoy's solution (60\% ethanol, $30 \%$ chloroform and $10 \%$ glacial acetic acid) or alike to treat surrounding structures. We suppose such a carcinogenic reagent should not be used for young patients like ours.

Although malignant transformation to squamous cell carcinoma is described as rare in the WHO book (Barnes et al. 2005), there are several reports on squamous cell carcinoma arising from KCOT (Maria et al. 2011; Tamgadge et al. 2013; Lukandu and Micha 2015). Close follow up is 


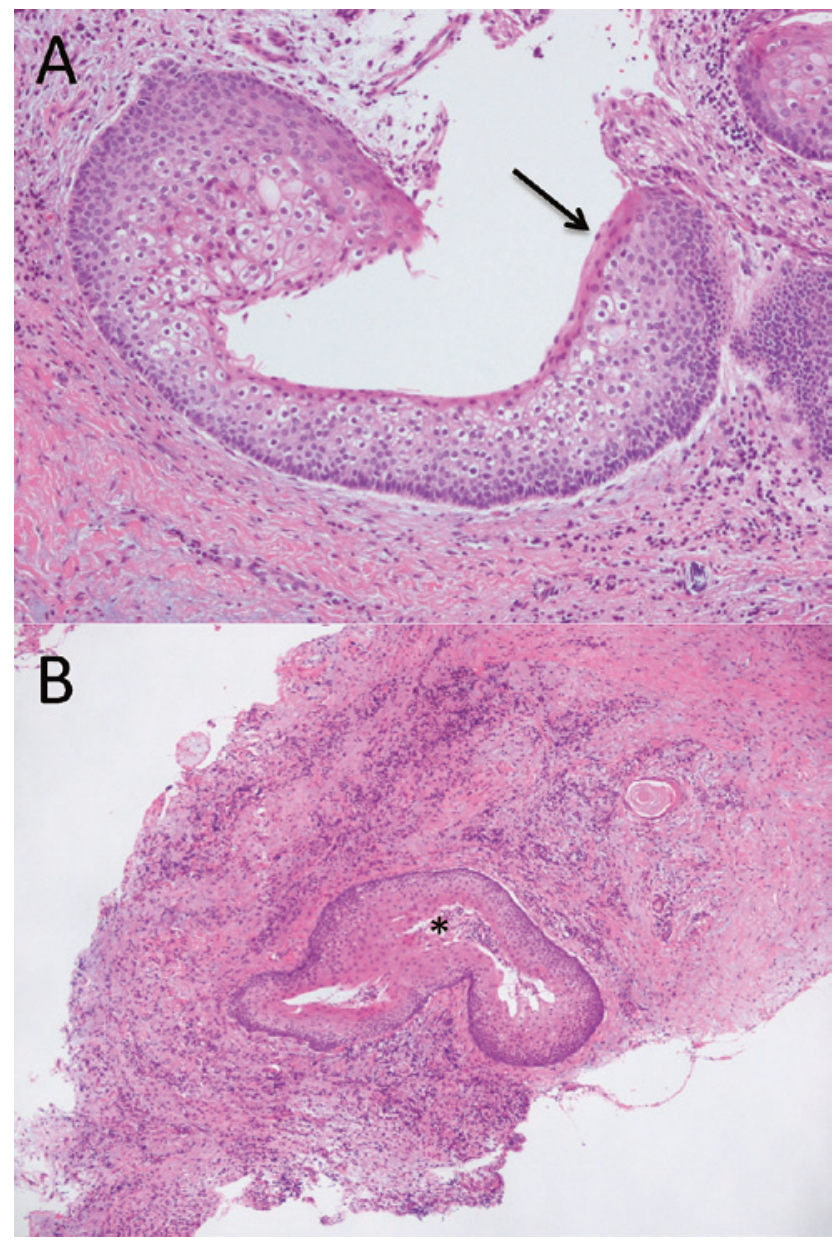

Fig. 3. Histopathological findings of the tumor (Hematoxylin and eosin staining).

(A) The cyst was lined by epithelial cells with parakeratosis (arrow). (B) Satellite cysts $(*)$ were seen with dense inflammatory cells within the fibrous stroma.

mandatory especially when complete resection is not guaranteed.

By using the Caldwell-Luc approach or endoscopic medial maxillectomy (EMM) approach, a wide surgical view can be obtained and straight instruments can be used (Wormald et al. 2003). When a tooth erupts from an easily accessible site, such as the medial wall of the maxillary sinus, or fragile bone, middle meatal antrostomy alone is appropriate for removal of the tooth (Hasbini et al. 2001; Di Pasquale and Shermetaro 2006). However, when a tooth erupts from an inaccessible site, such as the posterior, lateral or anterior wall of the maxillary sinus, and the tooth is firmly attached to the bone, it cannot be removed with middle meatal antrostomy, because torque does not transmit efficiently with curved instruments. Compared with the Caldwell-Luc approach, EMM is less invasive and can provide good visualization. The primary disadvantage of EMM is that it necessitates the removal of the inferior turbinate and nasolacrimal duct. Resection of the inferior turbinate might lead to empty nose syndrome. In patients who have had their inferior and the middle turbinate either partially or totally resected, a myriad of symptoms including nasal obstruction, crusting and dryness have been reported (Scheithauer 2010). Resection of the nasolacrimal duct might lead to epiphora and infection of lacrimal sac (Bartley 1992).

Recently, Nakayama et al. (2014) reported a case series of maxillary odontogenic cysts successfully treated with an EMMM approach. All the cases within their series were either cysts or odontogenic tumors confined to the maxillary sinus, i.e. not invading the hard palate. We believe that EMMM is the optimal approach to reduce damage to the surrounding structures during surgery and to avoid using the angled endoscope and curved instruments, which are not suitable for transmitting torque. EMMM provides access to the anterior wall of the maxillary sinus and is useful for inverted papilloma resection and odontogenic cysts including KCOT. Using EMMM, the posterior maxillary wall can be visualized at the center of the 0 degree endoscope field of view. Straight instruments can be used with EMMM, and all structures including the inferior turbinate, lacrimal duct, and the membranous lateral wall of the inferior meatus are preserved.

If total resection is mandatory for any KCOT, patients with invasive KCOT such as our patient will have postoperative deformity. Total resection of the KCOT in our patient would have resulted in a large defect of the hard palate, necessitating the use of an oral prosthesis. To avoid such an invasive treatment for a benign disease, we performed marsupialization using the EMMM approach. This is the first study reporting the treatment of KCOT with marsupialization using the EMMM approach. With this approach, almost all parts of the maxillary sinus can be reached (Nakayama et al. 2012, 2014). In our patient, there was inflammation in the KCOT (Fig. 3) and the cyst wall had a tendency to expand. Through marsupialization, a drainage pathway can be created, leading to recession of the inflammation and the cyst wall (Nakamura et al. 2002; Pogrel and Jordan 2004; Maurette et al. 2006). Combining the two concepts of EMMM and marsupialization, we have developed a minimally invasive treatment option for KCOT. If the tumor should grow during follow up, en bloc resection with external incision can be done as a staged operation.

\section{Conclusion}

The trans-nasal approach with EMMM is a direct, and minimally invasive method that can provide a direct field of view for maxillary KCOT treatment. This approach is especially useful for cases of maxillary KCOT where a tooth cannot be removed through middle meatal antrostomy. In cases where the KCOT has invaded the hard palate, marsupialization and close follow up may be a good option. By using a combination of the EMMM approach and marsupialization, even invasive KCOTs in the maxillary sinus can be treated in a minimally invasive way. If the 


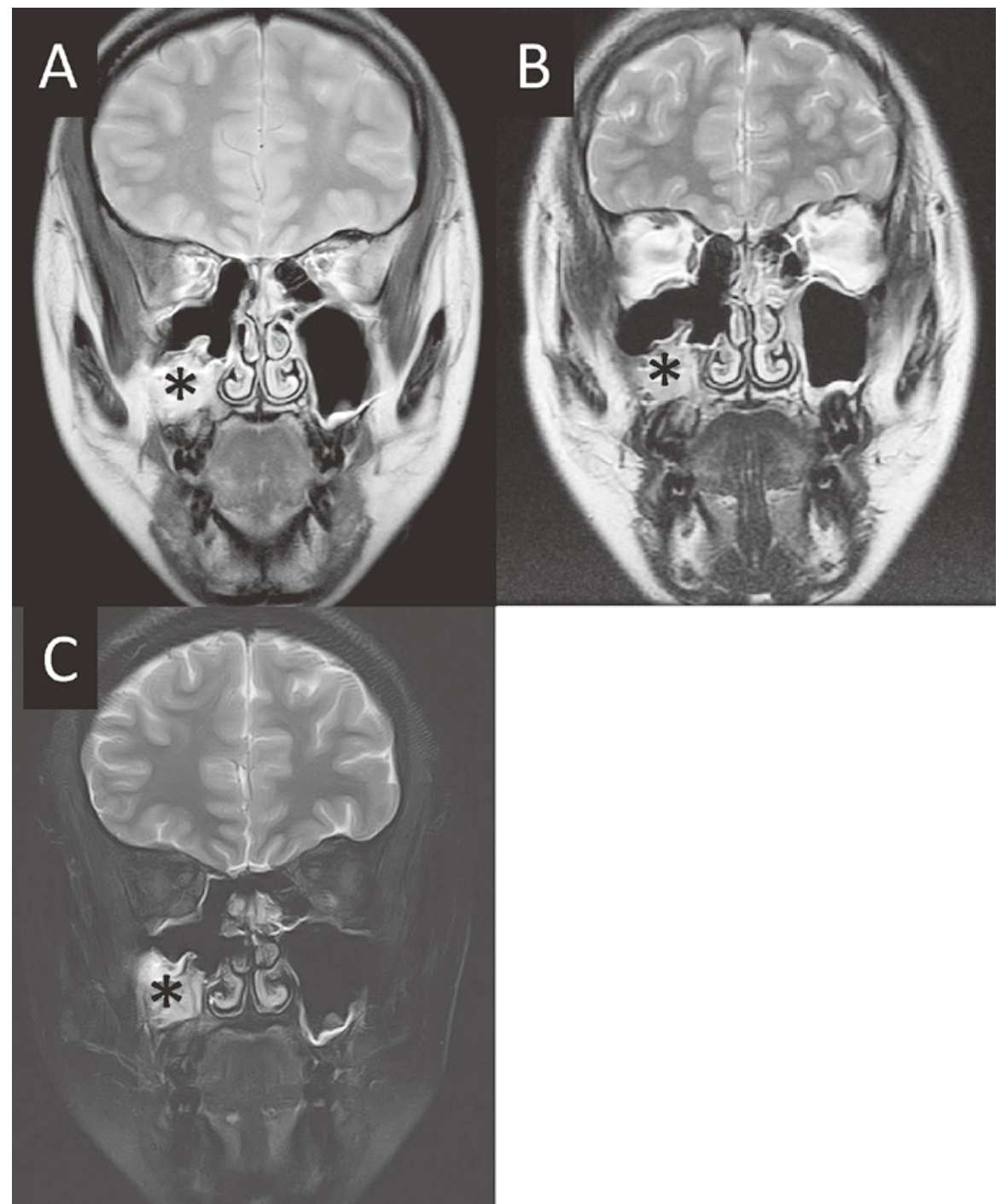

Fig. 4. Follow up T2-weighted MRI after operation.

(A) Four months after operation. High intensity lesion $\left(^{*}\right)$ is seen at the floor of the right maxillary sinus. (B) Seven months after operation. The size of the lesion $\left(^{*}\right)$ is unchanged. (C) Thirteen months after operation. The size of the lesion (*) is unchanged.

tumor should grow during post-operative follow up, en bloc resection can be performed to shrink the KCOT.

\section{Conflict of Interest}

The authors declare no conflict of interest.

\section{References}

Barnes, L., Eveson, J.W., Reichart, P. \& Sidransky, D. (2005) World Health Organization classification of tumours. Pathology and genetics of head and neck tumours, IARC Press, Lyon.

Bartley, G.B. (1992) Acquired lacrimal drainage obstruction: an etiologic classification system, case reports, and a review of the literature. Part 1. Ophthal. Plast. Reconstr. Surg., 8, 237-242.

Bataineh, A.B. \& al Qudah, M. (1998) Treatment of mandibular odontogenic keratocysts. Oral Surg. Oral Med. Oral Pathol. Oral Radiol. Endod., 86, 42-47.

Cakur, B., Miloglu, O., Yolcu, U., Göregen, M. \& Gürsan, N.
(2008) Keratocystic odontogenic tumor invading the right maxillary sinus: a case report. J. Oral Sci., 50, 345-349.

Di Pasquale, P. \& Shermetaro, C. (2006) Endoscopic removal of a dentigerous cyst producing unilateral maxillary sinus opacification on computed tomography. Ear Nose Throat J., 85, 747-748.

Giuliani, M., Grossi, G.B., Lajolo, C., Bisceglia, M. \& Herb, K.E. (2006) Conservative management of a large odontogenic keratocyst: report of a case and review of the literature. $J$. Oral Maxillofac. Surg., 64, 308-316.

González-Alva, P., Tanaka, A., Oku, Y., Yoshizawa, D., Itoh, S., Sakashita, H., Ide, F., Tajima, Y. \& Kusama, K. (2008) Keratocystic odontogenic tumor: a retrospective study of 183 cases. J. Oral Sci., 50, 205-212.

Grasmuck, E.A. \& Nelson, B.L. (2010) Keratocystic odontogenic tumor. Head Neck Pathol., 4, 94-96.

Habibi, A., Saghravanian, N., Habibi, M., Mellati, E. \& Habibi, M. (2007) Keratocystic odontogenic tumor: a 10-year retrospective study of 83 cases in an Iranian population. J. Oral Sci., 49, 229-235.

Hasbini, A.S., Hadi, U. \& Ghafari, J. (2001) Endoscopic removal 
of an ectopic third molar obstructing the osteomeatal complex. Ear Nose Throat J., 80, 667-670.

Lukandu, O.M. \& Micha, C.S. (2015) Primary intraosseous squamous cell carcinoma arising from keratocystic odontogenic tumor. Oral Surg. Oral Med. Oral Pathol. Oral Radiol., 120, e204-e209.

Maria, A., Sharma, Y. \& Chhabria, A. (2011) Squamous cell carcinoma in a maxillary odontogenic keratocyst: a rare entity. Natl. J. Maxillofac. Surg., 2, 214-218.

Maurette, P.E., Jorge, J. \& de Moraes, M. (2006) Conservative treatment protocol of odontogenic keratocyst: a preliminary study. J. Oral Maxillofac. Surg., 64, 379-383.

Menon, S. (2015) Keratocystic odontogenic tumours: etiology, pathogenesis and treatment revisited. J. Maxillofac. Oral Surg., 14, 541-547.

Nakamura, N., Mitsuyasu, T., Mitsuyasu, Y., Taketomi, T., Higuchi, Y. \& Ohishi, M. (2002) Marsupialization for odontogenic keratocysts: long-term follow-up analysis of the effects and changes in growth characteristics. Oral Surg. Oral Med. Oral Pathol. Oral Radiol. Endod., 94, 543-553.

Nakayama, T., Asaka, D., Okushi, T., Yoshikawa, M., Moriyama, H. \& Otori, N. (2012) Endoscopic medial maxillectomy with preservation of inferior turbinate and nasolacrimal duct. Am. J. Rhinol. Allergy, 26, 405-408.
Nakayama, T., Otori, N., Asaka, D., Okushi, T. \& Haruna, S. (2014) Endoscopic modified medial maxillectomy for odontogenic cysts and tumours. Rhinology, 52, 376-380.

Ohki, M. (2012) Transnasal marsupialization using endoscopic sinus surgery for treatment of keratocystic odontogenic tumor in maxillary sinus. Case Rep. Otolaryngol., 2012, 281402.

Pogrel, A.M. \& Jordan, R.C. (2004) Marsupialization as a definitive treatment for the odontogenic keratocyst. J. Oral Maxillofac. Surg., 62, 651-655.

Scheithauer, M.O. (2010) Surgery of the turbinates and "empty nose" syndrome. GMS Curr. Top. Otorhinolaryngol. Head Neck Surg., 9, Doc03.

Tamgadge, S., Tamgadge, A., Modak, N. \& Bhalerao, S. (2013) Primary intraosseous squamous cell carcinoma arising from an odontogenic keratocyst: a case report and literature review. Ecancermedicalscience, 7, 316.

Vered, M., Shohat, I., Buchner, A. \& Dayan, D. (2005) Myofibroblasts in stroma of odontogenic cysts and tumors can contribute to variations in the biological behavior of lesions. Oral. Oncol., 41, 1028-1033.

Wormald, P.J., Ooi, E., van Hasselt, C.A. \& Nair, S. (2003) Endoscopic removal of sinonasal inverted papilloma including endoscopic medial maxillectomy. Laryngoscope, 113, 867-873. 